\title{
THE IMPORTANCE OF NEUROIMAGING METHODS IN THE DIAGNOSIS OF EPILEPSY IN YOUNG MEN: LITERATURE REVIEW AND OWN CLINICAL CASE
}

\author{
Anna Voitiuk \\ Department of Neurology and Child Neurology \\ Kharkiv Medical Academy of Postgraduate Education \\ 58, Amosova str., Kharkiv, Ukraine, 61176 \\ Medical Centre «Neuron» \\ 4, M. Rybalko str., Kharkiv, Ukraine, 61091 \\ a_vojtyuk@yahoo.com
}

\begin{abstract}
Lifetime visualization of structural morphological changes of the brain in patients suffering from epilepsy and epileptic syndromes has become possible due to the introduction of neuroimaging methods into everyday medical practice. At the same time, neuroimaging is the main method of determining the etiology of epilepsy and its taxonomic position. One of these methods is magnetic resonance imaging (MRI). MRI is the method of choice in the examination of patients with diseases associated with brain development abnormalities.

The aim of this study is to consider the importance of using neuroimaging methods in this clinical case of epilepsy in a young person, taking into account complaints, anamnesis of the disease and life, neurological status, laboratory results for the correct diagnosis.

Conclusion. The presented clinical case of symptomatic epilepsy with focal vegetative-sensory seizures of medium frequency due to mass formation of the medial part of the right temporal lobe demonstrates the need and importance of a comprehensive examination using neuroimaging methods to clarify the etiology of the disease. Thus, magnetic resonance imaging has a high diagnostic value.

This article discusses the importance of MRI for the diagnosis of epilepsy and its etiology in a young man.

Keywords: epilepsy, seizures, electroencephalography, magnetic resonance imaging, dysembryoplastic neuroepithelial tumour, young men.
\end{abstract}

\section{Introduction}

The term «epilepsy» (epilepsis, epilepsia) was found for the first in the writings of Hippocrates as a definition of a special brain disease manifested by epileptic seizures [1]. Epilepsy is an independent nosological unit, significantly different in etiology and pathogenesis from acute cerebral pathology and focal diseases of the brain, which have epileptic seizures in their clinical picture [1,2].

The diagnosis of a first seizure or epilepsy syndrome is challenging and can therefore be misdiagnosed. Studies carried out in various settings have shown that the rate of misdiagnosis ranges from $4.6 \%$ to $30 \%$ [3].

The definition accurately reflects the essence of the disease and a number of important provisions. It is necessary to emphasize that the attacks should be spontaneous, and not provoked. The following factors can act as provoked:

- any exogenous intoxication (often alcohol);

- endogenous intoxication (decompensated ketoacidosis, uremia, liver failure, cancer intoxication);

- hyperthermia;

- somatic and neurological diseases (stroke, traumatic brain injury (TBI), brain tumour, etc.).

However, pathological conditions that were initially factors provoking seizures can become the basis for the formation of a structural and functional brain defect in the future, abnormal neurons of which will become a source of epileptogenesis and the formation of symptomatic epilepsy $[1,3]$.

Symptomatic focal epilepsy among patients is the absolute majority up to $71 \%$. In symptomatic focal epilepsy, the most common etiological factors of the disease are brain dysgenesis (focal cortical dysplasia, heterotopia, schizencephaly, pachygyria, etc.), mesial temporal sclerosis, brain tumours, vascular malformations, hereditary systemic diseases (tuberous sclerosis, encephalotri- 
geminal angiomatosis, etc.), trauma, Rasmussen syndrome, viral, infectious and parasitic diseases, metabolic disorders [2, 3].

One of the causes of epilepsy, symptomatic, is the presence of a tumour, for example, dysembryoplastic neuroepithelial tumour.

Dysembryoplastic neuroepithelial tumour (DNET) is a recently described, morphologically unique low-grade brain tumour. DNET is included in WHO 2007 classification as neuronal and mixed neuronal-glial tumour. Incidence among all neuroepithelial tumours is $1.2 \%$ in patients under 20 years and $0.2 \%$ in age over 20 years [4, 5]. Clinically these tumours are associated with focal seizures beginning before the age of 20 years, with no neurologic deficit and no stigmata of phacomatosis. It is a rare brain tumour not to be misdiagnosed. A multicystic appearance on MR images is a characteristic feature of DNET and corresponds to its myxoid matrix and multinodular architecture [6]. This feature is rare in gangliogliomas and glioneuronal malformations, and, as such, may help differentiate DNETs from these disorders. However, it may masquerade aggressive neural tumours such as oligodendroglioma, oligoastrocytoma, pilocytic astrocytoma, and ganglioglioma. Thus, magnetic resonance imaging has a high diagnostic significance [7].

The aim of the study. To consider the importance of using neuroimaging methods in this clinical case of epilepsy in a young person, taking into account complaints, anamnesis of the disease and life, neurological status, laboratory results for the correct diagnosis.

\section{Case report \\ Ethical aspects}

The work complies with the ethical standards of the Helsinki Declaration of the World Medical Association. A written informed consent was obtained from all participants and the study was approved by the Bioethics Committee of Kharkiv Medical Academy of Postgraduate Education (report No. 2 of $16^{\text {th }}$ March 2021).

Patient P., 31 years old (01.09.1989), was admitted on 03/01/2021 to the Kharkiv Railway Clinical Hospital No. 1 of Branch of «HC» JSC «Ukrzaliznytsia» with complaints of periodic headache, dizziness, stereotypical paroxysmal states lasting up to 10-20 seconds, which begin with the smell of ozone, a feeling of heart sinking or palpitations, a feeling of lack of air, an irresistible desire to sleep, periodically increasing blood pressure to $145 / 90 \mathrm{~mm} \mathrm{Hg}$, tension and pain in the cervical spine, which increases when the head turns to the sides.

From the anamnesis of the disease, it is known that headaches periodically bother the patient from the age of 5-6 years, as a result of which an ambulance was repeatedly called. The treatment was prescribed for migraine cephalalgias.

For the first time, a seizure of consciousness's loss in a young man developed at the age of 8 (1997) to a painful reaction (arm fracture). The attack was repeated in 2010, when in the morning after urinating he lost the consciousness, convulsions, and there was not bite of the tongue. He did not seek medical help on this matter. In the spring of 2014, the patient felt a sharp pain in the cervical spine in the morning after getting out of bed. In the infirmary, during the injection of movalis, the patient lost the consciousness, fell; there were no convulsions, urination. According to the patient, it is known that a decrease in blood pressure to $60 / 40 \mathrm{~mm} \mathrm{Hg}$ was recorded. With outside help, he was transferred to the couch, where he again lost consciousness without convulsions, tongue bite and amnesia. The young man had pallor of the skin, hyperhidrosis. A few months later (09/26/2014) after waking up, the patient again felt a sharp pain in the cervical spine, got up, lost consciousness without convulsions, tongue bite and amnesia. I turned to the medical center, as I was on vacation in Turkey. After a few minutes, dizziness, weakness, drowsiness, and fainting appeared again. The patient was taken to the hospital, an X-ray of the cervical spine was made, manual therapy was performed. The medical documentation is not provided. Since 2014, he has been repeatedly treated at the Kharkiv Railway Clinical Hospital No. 1 of Branch of «HC» JSC «Ukrzaliznytsia» with the diagnosis: «Cervicalgia due to osteochondrosis C4-C5, C5-C6, spondyloarthrosis, uncovertebral arthrosis C4-C5, C5-C6, wedge-shaped deformity of the body C6. The vegetative-vascular dystonia of the mixed type with syncopal (vasovagal) paroxysms in the anamnesis»». 
This patient has been experiencing deterioration since September 2020, when shortterm (10-20 seconds), stereotypical seizures with the smell of ozone, lack of air, an irresistible desire to sleep, recurring 1 time every 1-2 months, appeared. According to the patient, up to 10 similar paroxysms per day were observed on 02/25/2021. Since December 2020, he has been disturbed by sleep attacks several times, when he woke up due to a feeling of lack of air, breathed noisily, felt an irresistible desire to sleep, after 10-20 seconds the attacks stopped, fell asleep. The pain and tension in the cervical spine increased for 2 days ago. Due to the deterioration of his health on $03 / 01 / 2021$, the young man was hospitalized.

It is known from the anamnesis vitae that contacts with persons with fever, respiratory diseases, clinically healthy persons who were in epidemiologically unfavourable regions with a high prevalence of patients and infected with COVID-19 over the past 2 weeks are denied. The patient denies signs of respiratory diseases, an increase in body temperature over the past 2 weeks. He denies tuberculosis, viral hepatitis, sexually transmitted diseases, diabetes mellitus, allergic reactions to medications.

At the time of examination, the general condition of the patient is satisfactory. The right physique. The skin and visible mucous membranes are clean, of the usual colour. The breathing is vesicular, there are not wheezes. The heart tones are rhythmic. AT 130/90 $\mathrm{mmHg}$, heart rate 72 beats $/ \mathrm{min}$. The abdomen is soft, painless with palpation. There is not swelling. The physiological functions are controlled.

The level of consciousness is clear, oriented correctly in time and place, intelligence corresponds to age in the neurological status. Available to productive contact in full. The meningeal signs are negative. The eye slits $\mathrm{D} \geq \mathrm{S}$, pupils $\mathrm{D}=\mathrm{S}$. photoreaction of average liveliness. The movements of the eyeballs are possible in full. The convergence is weakened. There is not nystagmus. The nasolabial folds are symmetrical. The pharyngeal reflex is triggered, swallowing is not disturbed. The tongue is moist, not overlaid, along the middle line. The speech is not broken. There are not bulbar violations. The muscle tone is preserved. The muscle strength is sufficient. Tendon and periosteal reflexes from the hands $\mathrm{D}=\mathrm{S}$, knee $\mathrm{D}=\mathrm{S}$, Achilles $\mathrm{D} \leq \mathrm{S}$, medium vivacity. There are not pathological stop signs. Sensitive disorders are not detected. The finger and knee-heel tests are performed satisfactorily. The slight rocking in the Romberg's pose. The coordination tests are performed satisfactorily. The pronounced tension of the paravertebral muscles at the cervical level, painful palpation of the paravertebral points at this level.

During the entire period of stay in the hospital, the following studies were conducted:

There were not significant deviations from the reference values in general clinical blood and urine tests.

Chest X-ray (03/01/2021): without features.

ECG (03/02/2021): sinus rhythm, correct. Semi-vertical electrical position of the heart.

EEG (03/01/2021): slight diffuse EEG changes with signs of rhythm disorganization and single flashes of pointed waves in the frontotemporal regions on the right (Fig. 1).

When conducting a hyperventilation test, a short-term bilateral synchronous discharge of spike activity is recorded [8] (Fig. 2).

EEG with sleep deprivation (03/02/2021): mild diffuse EEG changes with single flashes of pointed waves in the frontotemporal regions, mainly on the right. During the hyperventilation test, dizziness, ringing in the ears and discomfort behind the sternum. The test is suspended. It is not significant changes in the rhythm were detected [9] (Fig. 3).

Considering the presence of epileptic seizures over the past six months, the diagnosis was made: «Cryptogenic epilepsy with focal vegeto-sensory seizures of medium frequency. Volumetric formation of the medial part of the right temporal lobe. Cervicalgia due to osteochondrosis C4-C5, C5-C6, spondyloarthrosis, with intervertebral disc protrusion with canal stenosis in the C5-C6 segment. The vegetative-vascular dystonia of a mixed type with syncopal (vaso-vagal) paroxysms in the anamnesis» [10]. For diagnostic purposes, the patient was referred for magnetic resonance imaging of the brain.

Magnetic resonance imaging of the brain (1.5 Tesla) (03/03/2021): MR-signs of structural changes in the medial part of the right temporal lobe [11] (DNET - dysembrioplastic neuroepithelial tumour) [5]. The cyst of the right maxillary sinus (Fig. 4). A consultation with a neurosurgeon, observation in dynamics is recommended. 
00:04:48 фотостинупячия 2 гu

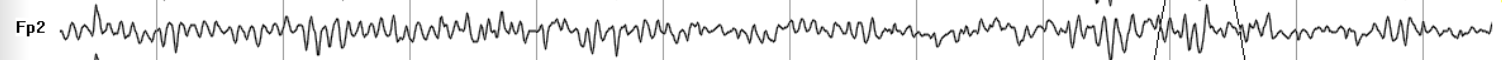

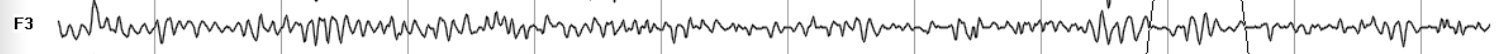

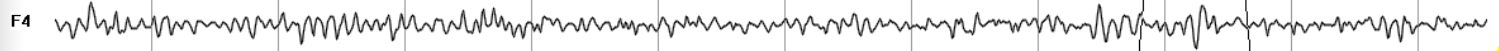

F7 Connm

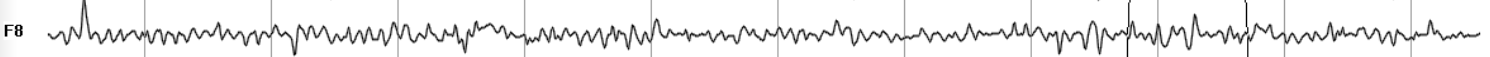
т3 CL,

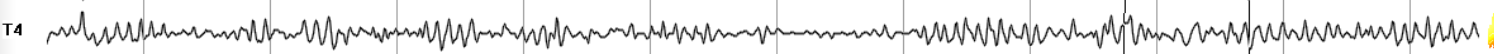

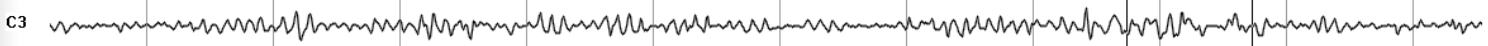

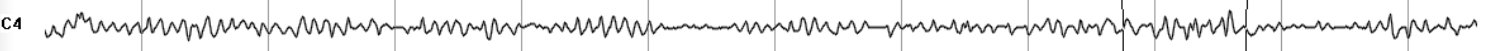

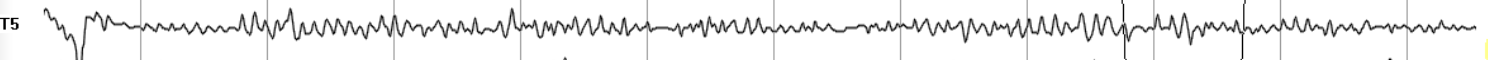

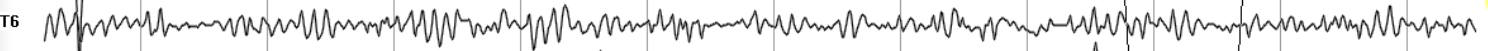

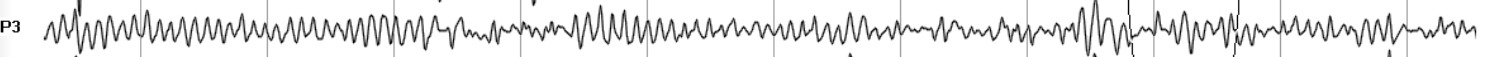

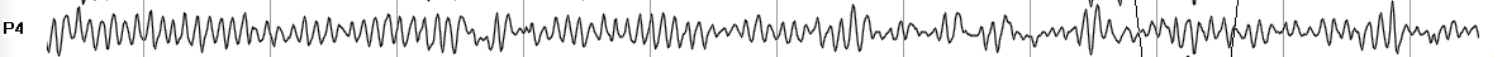

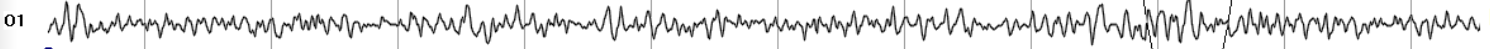

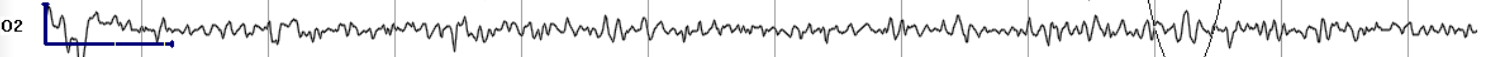

Fig. 1. The EEG fragment of patient P., 31 y. o. Gain $50 \mathrm{mv} / 10 \mathrm{~mm}$, sweep speed $30 \mathrm{~mm} / \mathrm{sec}$, high frequency $0.1 \mathrm{sec}$, low frequency $30 \mathrm{~Hz}$ (EEG scaled, $50 \mathrm{mv}, 1 \mathrm{sec}$ marked)

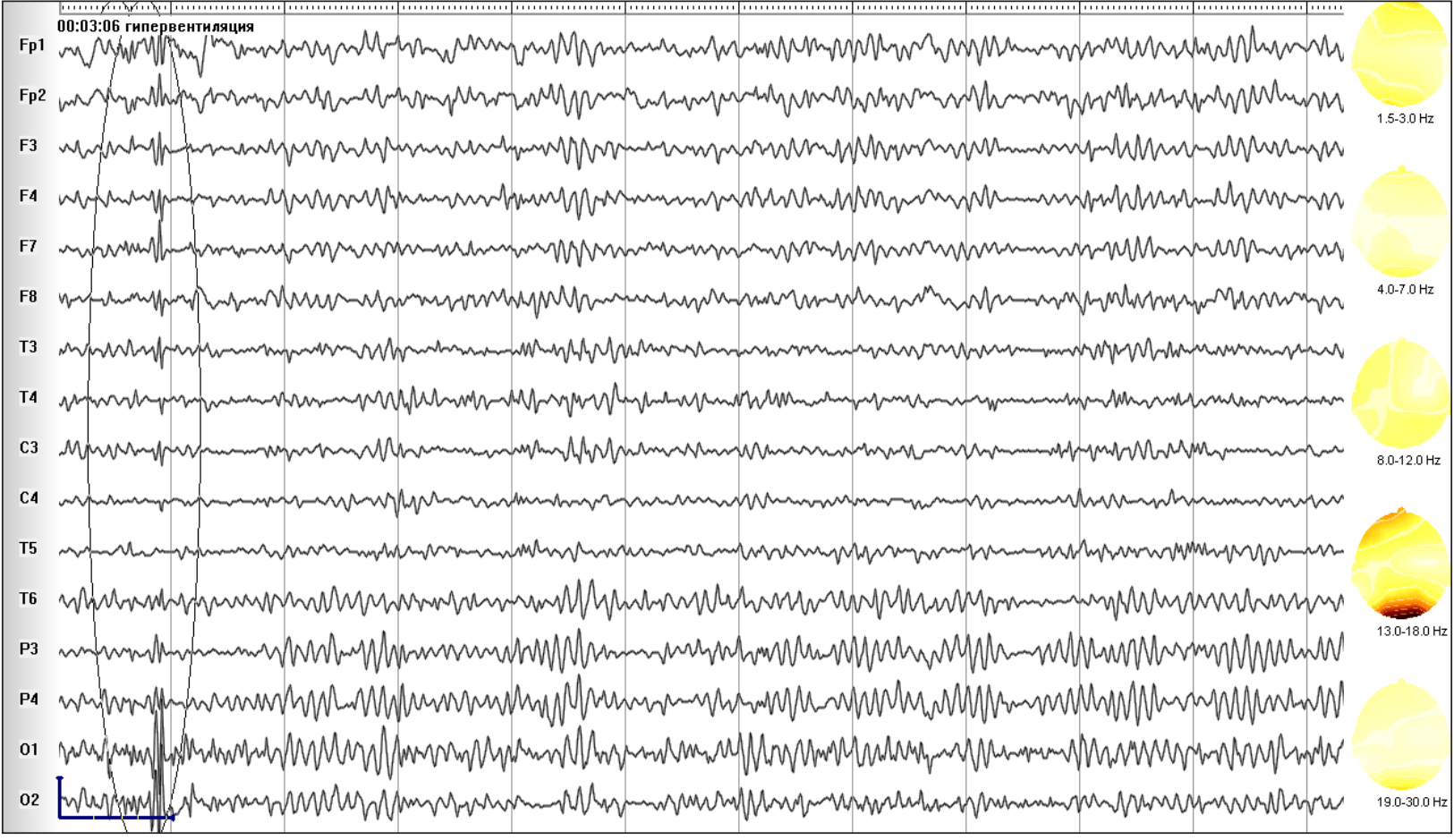

Fig. 2. The EEG fragment of patient P., 31 y. o. (hyperventilation test). Gain $50 \mathrm{mv} / 10 \mathrm{~mm}$, sweep speed $30 \mathrm{~mm} / \mathrm{sec}$, high frequency $0.1 \mathrm{sec}$, low frequency $30 \mathrm{~Hz}$ (EEG scaled, $50 \mathrm{mv}, 1 \mathrm{sec}$ marked)

Magnetic resonance imaging of the cervical spine (03/03/2021): MR-signs of osteochondrosis of the cervical spine with intervertebral disc protrusion, spinal canal stenosis in the C5-C6 segment. Spondyloarthrosis, uncovertebral arthrosis. Spondylosis [11, 12] (Fig. 5).

Consultation with an ophthalmologist (03/04/2021): the fundus is the norm. Arteries, veins are full-blooded, dilated. 


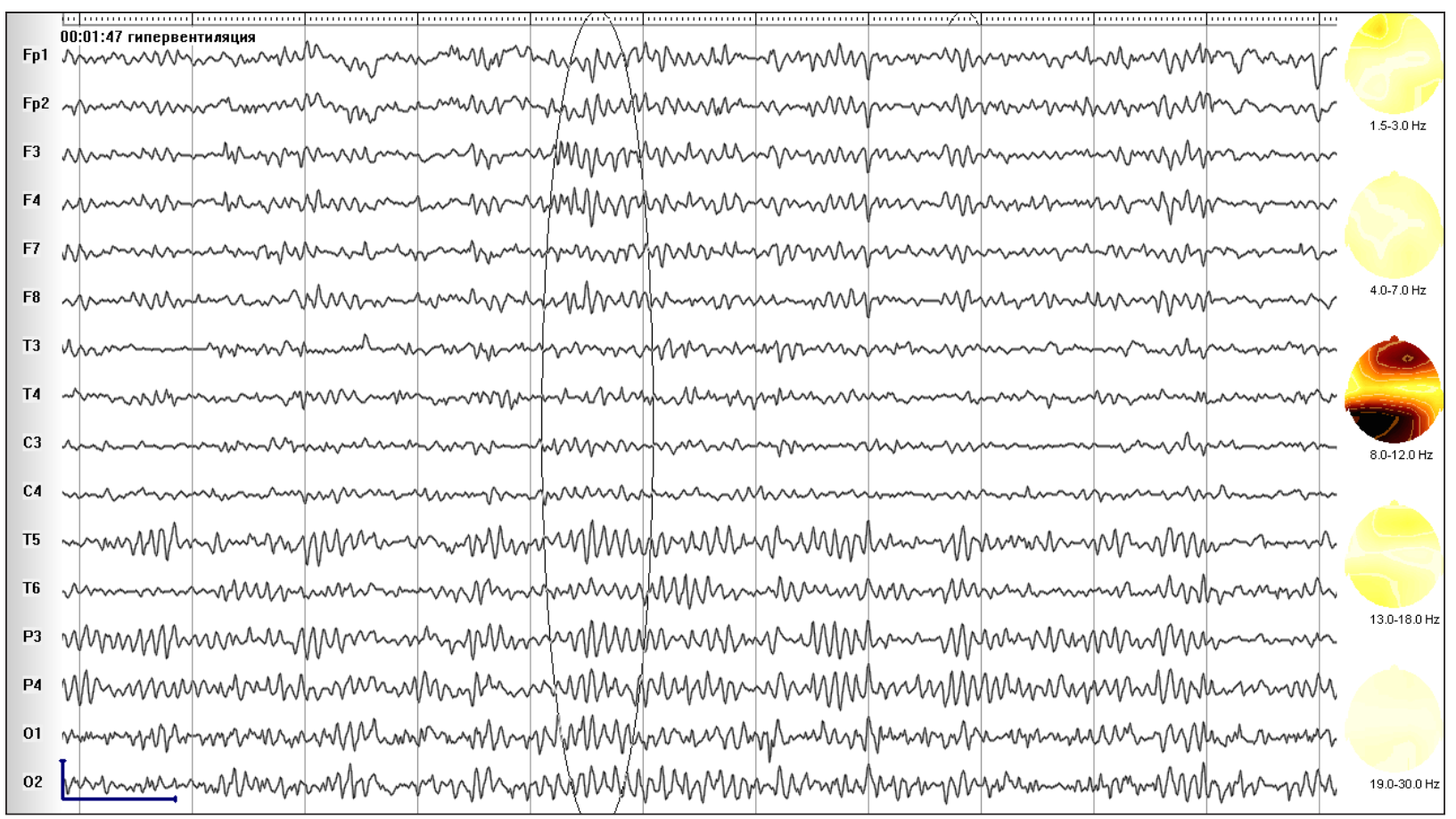

Fig. 3. The EEG fragment with sleep deprivation of patient P., 31 y. o. Gain $50 \mathrm{mv} / 10 \mathrm{~mm}$, sweep speed $30 \mathrm{~mm} / \mathrm{sec}$, high frequency $0.1 \mathrm{sec}$, low frequency $30 \mathrm{~Hz}$ (EEG scaled, $50 \mathrm{mv}, 1 \mathrm{sec}$ marked))
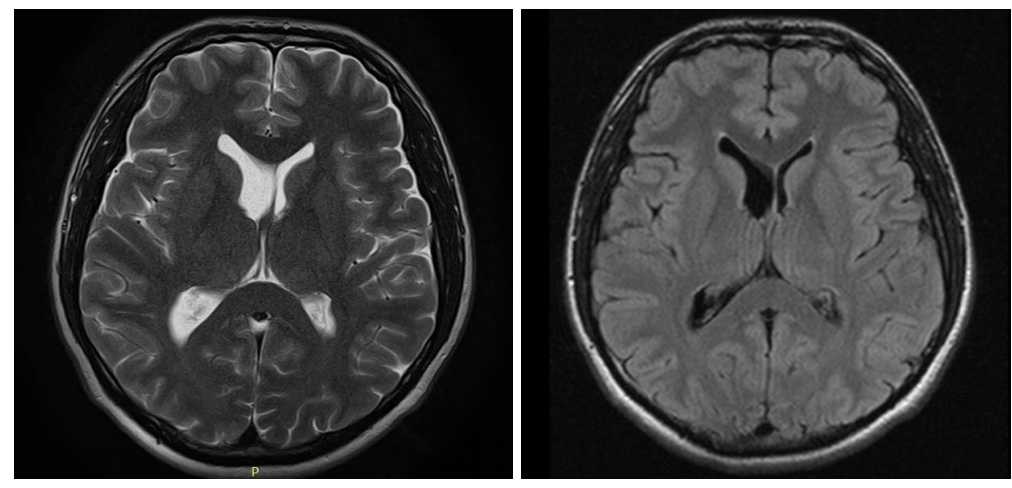

Fig. 4. MRI of the brain of patient P., 31 y. o.
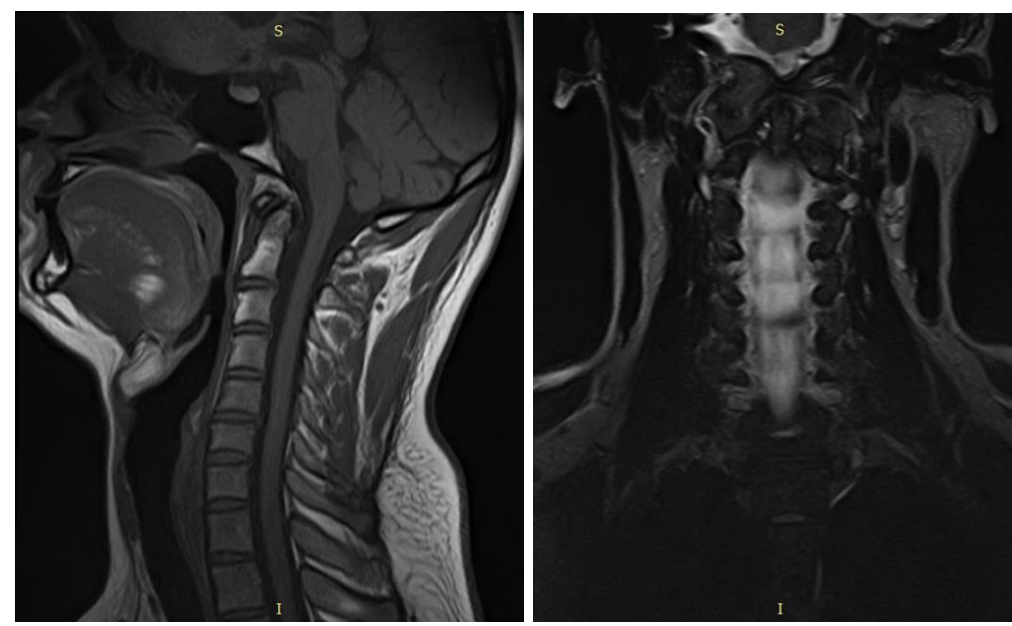

Fig. 5. MRI of the cervical spine of patient P., $31 \mathrm{y}$. o. 
Consultation of a neurosurgeon (03/04/2021): according to MRI datas, there is a volumetric formation of the medial part of the right temporal lobe. The surgical treatment is not indicated at the moment (given the small size, deep location and lack of significant growth). A consultation is needed in a neurosurgical clinic that operates with a cyber knife.

This patient was prescribed the following treatment: magnesium sulfate intravenously, tivortin intravenously, vitaxone intravenously, dexamethasone intravenously, omez $20 \mathrm{mg}$, keppra $500 \mathrm{mg} 2$ times a day.

During his stay in the hospital, the patient had not epileptic seizures. The headache and pain in the cervical spine decreased. However, the patient periodically complained of dizziness and shakiness. He was discharged from the Kharkiv Railway Clinical Hospital No. 1 of Branch of «HC» JSC «Ukrzaliznytsia» on the tenth day with the diagnosis: «Symptomatic epilepsy with focal vegeto-sensory seizures of medium frequency. Volumetric formation of the medial part of the right temporal lobe. Cervicalgia due to osteochondrosis $\mathrm{C} 4-\mathrm{C} 5, \mathrm{C} 5-\mathrm{C} 6$, spondyloarthrosis, with intervertebral disc protrusion with canal stenosis in the C5-C6 segment. The vegetative-vascular dystonia of a mixed type with syncopal (vaso-vagal) paroxysms in the anamnesis». Upon discharge from the hospital, the patient's well-being improved, compliance with medical and labour recommendations was suggested.

\section{Conclusions}

The presented clinical case of symptomatic epilepsy with focal vegetative-sensory seizures of medium frequency due to mass formation of the medial part of the right temporal lobe demonstrates the need and importance of a comprehensive examination using neuroimaging methods to clarify the etiology of the disease.

Thus, magnetic resonance imaging has a high diagnostic value.

MRI (magnetic resonance imaging) is the «gold» standard for diagnosing epilepsy. The method allows visualizing the morphological substrate of epilepsy, on the basis of which the clinician decides on the choice of the method of treatment (conservative or surgical).

High-resolution MRI reveals structural abnormalities in the anatomical structures of the brain responsible for the formation of pathological epileptic activity. These are changes that usually go unnoticed by a standard MRI.

\section{Conflict of interest}

The authors declare that they have no conflicts of interest.

\section{Financing}

The study was performed without financial support.

\section{Acknowledgments}

I express our gratitude to the patient who took part in the study, as well as the specialists who contribute to the investigation.

\section{References}

[1] Litovchenko, T. A. (2016). Epilepsy: terminology, epidemiology, classification, etiology, pathogenesis. NeuroNews, 2, $18-24$.

[2] Fisher, R. S., Acevedo, C., Arzimanoglou, A., Bogacz, A., Cross, J. H., Elger, C. E. et. al. (2014). ILAE Official Report: A practical clinical definition of epilepsy. Epilepsia, 55 (4), 475-482. doi: http://doi.org/10.1111/epi.12550

[3] Voitiuk, A. A. (2019). Epilepsy: Age and Gender Aspects. Archives in Neurology \& Neuroscience, 3 (4). doi: http://doi.org/ 10.33552/ann.2019.03.000570

[4] Sukheeja, D., Mehta, J. (2016). Dysembryoplastic neuroepithelial tumor: A rare brain tumor not to be misdiagnosed. Asian Journal of Neurosurgery, 11 (2), 174. doi: http://doi.org/10.4103/1793-5482.175643

[5] Luzzi, S., Elia, A., Del Maestro, M., Elbabaa, S. K., Carnevale, S., Guerrini, F. et. al. (2019). Dysembryoplastic Neuroepithelial Tumors: What You Need to Know. World Neurosurgery, 127, 255-265. doi: http://doi.org/10.1016/j.wneu.2019.04.056

[6] Sontowska, I., Matyja, E., Malejczyk, J., Grajkowska, W. (2017). Dysembryoplastic neuroepithelial tumour: insight into the pathology and pathogenesis. Folia Neuropathologica, 55 (1), 1-13. doi: http://doi.org/10.5114/fn.2017.66708 
[7] Siraj, F., Gupta, P., Malik, A., Shankar, K. (2021). Clinical and histopathological profile of dysembryoplastic neuroepithelial tumor: An experience from a tertiary care center. Journal of Cancer Research and Therapeutics, 17 (4), 912-916. doi: http:// doi.org/10.4103/jcrt.jcrt_632_19

[8] Zenkov, L. R. (2017). Klinicheskaia elektroentsefalografiia. S elementami epileptologii. Moscow: MEDpress-inform, 356.

[9] Rektor, I., Kuba, R., Brázdil, M. (2002). Interictal and Ictal EEG Activity in the Basal Ganglia: An SEEG Study in Patients with Temporal Lobe Epilepsy. Epilepsia, 43 (3), 253-262. doi: http://doi.org/10.1046/j.1528-1157.2002.28001.x

[10] International League Against Epilepsy. Available at: http://www.ilae.org

[11] Santos Armentia, E., Martín Noguerol, T., Suárez Vega, V. (2019). Técnicas avanzadas de resonancia magnética en patología tumoral de cabeza y cuello. Radiología, 61 (3), 191-203. doi: http://doi.org/10.1016/j.rx.2018.12.004

[12] Gröhn, O., Pitkänen, A. (2007). Magnetic Resonance Imaging in Animal Models of Epilepsy?Noninvasive Detection of Structural Alterations. Epilepsia, 48 (s4), 3-10. doi: http://doi.org/10.1111/j.1528-1167.2007.01236.x

Received date 14.12.2021

(C) The Author(s) 2022

Accepted date 20.01.2022

Published date 31.01.2022

This is an open access article under the Creative Commons CC BY license

How to cite: Voitiuk, A. (2022). The importance of neuroimaging methods in the diagnosis of epilepsy in young men: literature review and own clinical case. EUREKA: Health Sciences, 1, 56-62. doi: http://doi.org/10.21303/2504-5679.2022.002179 